\section{Das Komplementsystem: \\ Alter Hut oder Ziel neuer Therapieansätze?}

Zusammenfassung. Das Komplementsystem ist ein multifaktorielles Proteinkaskadensystem das im Zentrum der unspezifischen frühen Immunantwort steht. Seine wesentliche Aufgabe ist die Aktivierung zellulärer Abwehrmechanismen, die Opsonierung von Fremdkörpern und die Zerstörung entsprechender Zielzellen. Die Bedeutung der einzelnen Komplementkomponenten für die Bakterienelimination in der Sepsis wird jedoch immer noch kontrovers diskutiert. Trotz oder gerade wegen der effizienten zytotoxischen Wirkung kann die überschießende Aktivierung der Komplementkaskade im Organismus zu schweren lebensbedrohlichen Gewebeschäden führen. Eine Reihe von tierexperimentellen Studien hat gezeigt, daß genetische Komplementdefekte oder Komplement- Depletion den Verlauf von schweren Entzündungsvorgängen günstig beeinflussen und Organschäden reduzieren können. Versuche die Aktivierung der Komplementkaskade zu supprimieren sind einerseits die Applikation endogener Komplementinhibitoren z. B. C1-Inhibitor (C1-INH) oder rekombinanter Komplementrezeptoren wie des löslichen Komplementrezeptors 1 (rsCR1). Andererseits stellt die Gabe von Antikörpern gegen Schlüsselproteine (C3 oder $\mathrm{C5}$ ), deren Aktivierungsprodukte (C5a) oder gegen die Komplementrezeptor 3 (CR3, CD18/ 11b) -mediierte Adhäsion von Entzündungszellen an das vaskuläre Endothel effektive Möglichkeiten der Komplementmodulation dar. Darüber hinaus wird derzeit zur Vermeidung Komplement-vermittelter Abstoßungsreaktionen bei Xenotransplantation der Einbau von membranständigen Komplementregulatoren in das Spenderorgan (DAF- CD55, MCP- CD46 oder CD59) untersucht. Die beschriebenen Interventionen schützten in einer Vielzahl von tierexperimentellen Modellen von Sepsis, bei myokardialem und intestinalem Ischämie-/Reperfusionsschaden, ARDS, Nephritis und Transplantatabstoßung vor Komplement-mediierten Gewebeschäden. Gestützt auf neue klinische Daten könnte die Komplementinhibition eine geeignete therapeutische Strategie darstellen, überschießende Entzündungsvorgänge zu dämpfen. Eigene Untersuchungen zeigten in einem Modell Komplementinduzierter Lungenschädigung die Effizienz einer Modulation der Komplementkaskade durch die Komplementregulatoren C1-Inhibitor und löslichem Komplementrezeptor 1. Hingegen muß im Hinblick auf eine suffiziente Erregerabwehr der Einsatz von Komplementinhibitoren kritisch abgewogen werden.

Schlüsselwörter: Komplement - Anaphylatoxine - terminaler Komplement-Komplex - C-1INH - SCR-1 - MOF - SIRS

\author{
A. Heller, Thea Koch \\ Klinik und Poliklinik für Anaesthesiologie und Intensivmedizin \\ Universitätsklinikum Carl Gustav Carus \\ Technische Universität Dresden \\ (Direktor: Prof. Dr. med. D. M. Albrecht)
}

The complement system: An old story or target of new therapeutic approaches? The complement system is a multifactorial protein cascade system which is essentially involved in the early unspecific immune response. Its major function is the activation of cellular defense mechanisms, opsonisation of foreign particles and the destruction of target cells. While the impact of the different complement components for bacterial elimination still remains controversial, overwhelming activation of the complement cascade, however, can induce life threatening tissue damage due to the effective cytotoxic properties. In the last years a variety of studies demonstrated beneficial, organ protective effects of complement modulation in models of severe inflammation. Attempts to control the complement system include the application of endogenous complement inhibitors e.g. C1-inhibitior (C1-INH) or the administration of recombinant complement receptors such as the soluble complement receptor 1 (rsCR1). Moreover antibodies against key proteins $(C 3, C 5)$, against their activation products (C5a) or against complement receptor 3 (CR3, CD18/ 11b) mediated adhesion of leukocytes to the vascular endothelium, represent effective options of complement modulation. Besides this, insertion of membrane bound human complement regulators (DAF- CD55, MCP- CD46 or CD59) into xenogenic donor organs has proven effectiveness to prevent xenograft rejection. The described interventions protected from severe organ damage in various animal models of sepsis, myocardial and intestinal ischaemiareperfusion injury, ARDS, nephritis, and xenograft rejection. With respect to recent clinical data, complement inhibition could represent a useful therapeutic strategy to control overwhelming inflammation. Own experiments demonstrated protective effects of complement modulation with $\mathrm{C} 1 \mathrm{INH}$ and rsCR1 in a model of complement induced pulmonary injury. With respect to sufficient host defense, however, the use of complement inhibitors must be considered carefully.

Key words: Complement - anaphylatoxins - terminal complement complex - C-1INH - SCR-1 - ARDS - MOF - SIRS

\section{Einführung}

Entzündungen und Traumata setzen den Organismus durch Freisetzung pro-inflammatorischer Mediatoren einer erhöhten Gefahr von Infektionen oder Sekundärschäden aus, die zu isoliertem oder multiplem Organversagen führen können. Dabei erfolgt die Aktivierung zellulärer und humoraler Kaskadensysteme durch eine Vielzahl von Stimuli [1,2]. Aufgrund der engen Verflechtung der einzelnen Kaskadensysteme mitein- 
ander kann die Induktion eines Systems weitere proinflammatorische Kaskaden anstoßen und damit den Entzündungsvorgang perpetuieren. Der diagnostische Wert von Komplementaktivierungsprodukten als Parameter für die Voraussage der Entwicklung von ARDS, Sepsis oder dem klinischen Outcome wurde in einigen klinischen Studien beschrieben $[3,4]$.

\section{Funktionsweise des Komplementsystems}

Das Komplementsystem stellt eine schnelle effektive Barriere des Organismus gegen eindringende Mikroorganismen dar $[5,6]$. Durch seine verschiedenen biologischen Aktivitäten ist das Komplementsystem ein inflammatorisches Schlüsselmediatorsystem, eine natürliche Antwort des Organismus auf jede Art der Durchbrechung der Körperintegrität (Tab.1). Daneben scheint das Komplementsystem an der Regulation von Immunfunktionen beteiligt zu sein. Das Komplementsystem besteht aus ca. 30 Proteinen, die in einer kaskadenartigen Reaktionssequenz als Kontrollproteine oder als zelluläre Rezeptoren wirken (Abb.1). Die Aktivierung dieser Sequenz kann auf dre verschiedenen Wegen erfolgen. Entweder Antikörper-abhängig (klassischer Weg), durch Fremdoberflächen getriggert (alternativer Weg) oder über den erst vor kurzem entdeckten MBL/ MASP-Weg (mannan binding lectin/ MBL-associated serine protease) [7].

Im Verlauf der Komplementkaskade wird neben den Anaphylatoxinen C3a und C5a der „membrane attack complex“ C5b-9 gebildet. $\mathrm{C} 3 \mathrm{a}$ und $\mathrm{C} 5 \mathrm{a}$ fungieren hierbei als pro-inflammatori-

Tab.1 Zusammenfassung der pro-inflammatorischen Wirkungen von Komplement

1. Chemotaxis und Aktivierung von neutrophilen Granulozyten (C3a und $\mathrm{C} 5 \mathrm{a}$ )

2. Opsonisierung zur Phagozytoseerleichterung von Bakterien (C3)

3. Mastzelldegranulation (C3a und $\mathrm{C} 5 \mathrm{a}$ )

4. Lyse von Bakterien und Fremdzellen (C5b-9)

5. Kontraktion von glatten Muskelzellen (C3a und $\mathrm{C} 5 \mathrm{a}$ )

6. Erhöhte Gefäßpermeabilität (C3a und C5a)

7. Komplexbildung in den Keimzentren (C3) sche Aktivatoren. Sie induzieren Chemotaxis von Leukozyten, Degranulation von Phagozyten, Mastzellen und Basophilen. Darüber hinaus kommt es anaphylatoxinvermittelt zur Kontraktion von glatter Muskulatur und erhöhter Gefäßpermeabilität [8]. Am Ende der Komplementsequenz steht die Polymerisierung von C9- Molekülen zu einem röhrenförmigen Membranangriffskomplex, der eine Pore in der Zielzellmembran erzeugt. Durch den so entstandenen Kanal können Ionen, Wasser und Enzyme in das Innere der Zielzelle eindringen und diese zerstören.

Neben den Aufgaben, die das Komplementsystem als Teil der angeborenen humoralen Immunabwehr wahrnimmt kommt ihm auch im Rahmen der adaptiven Immunität eine wichtige Bedeutung zu. Verschiedene immunkompetente Zellen (z. B. Monozyten, Makrophagen, Neutrophile oder B-Lymphozyten) tragen Komplementrezeptoren (CR) auf ihrer Oberfläche. CR-1 und CR-3 spielen in diesem Zusammenhang eine wesentliche Rolle für die Initiierung der Phagozytose von Bakterien. CR-2 findet sich vorwiegend auf B-Lymphozyten und trägt als Teil des B-Zell-CD19-Korezeptorkomplexes zur B-Zellaktivierung durch Antigene bei [9].

Die Bedeutung der einzelnen Komplementkomponenten für die bakterielle Abtötung in der Sepsis wird noch immer kontrovers diskutiert. Wir haben daher in einer eigenen Untersuchung an C6-defekten Kaninchen die Effekte der Anaphylatoxine (C3a, C5a) und des terminalen Komplementkomplexes (C5b-9) bei der Endotoxinämie differenziert und die Auswirkungen des C6-Defektes auf die Bakterienelimination, Phagozytose- und Burstkapazität von neutrophilen Granulozyten (PMN) untersucht [10]. Zur Quantifizierung des Clearance-Prozesses wurde anästhesierten Kaninchen mit genetischem C6-Defekt ( $\mathrm{n}=7$ ) Endotoxin (LPS; $40 \mu \mathrm{g} / \mathrm{kg} / \mathrm{h}$ ) über $4 \mathrm{~h}$ infundiert. Diese Tiere sind nicht in der Lage den terminalen Komplementkomplex $\mathrm{zu}$ bilden. $1 \mathrm{~h}$ nach Beginn der LPSInfusion wurde eine definierte Anzahl Escherichia (E.) coli $\left(10^{8} \mathrm{CFU}\right)$ intravenös injiziert. Als Kontrollen dienten 7 Kaninchen ohne Komplementdefekt. In kurzen Zeitintervallen wurden Blutproben zur Bestimmung der PMN-Funktion, sowie für bakteriologische Kulturen entnommen. Hierdurch konnten wir

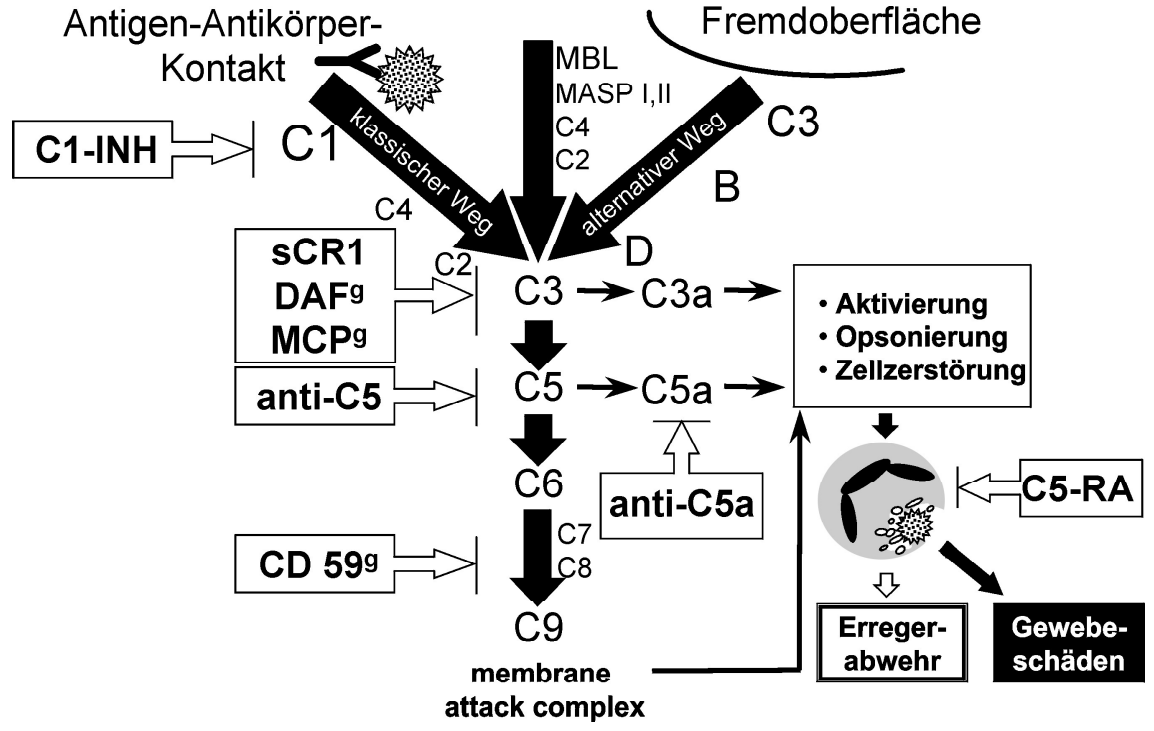

Abb.1 Komplementsystem mit Aktivierungswegen und therapeutischen Interventionsmöglichkeiten. Anti-Komplementfaktoren Strategien: C1-Inhibitor (C1-INH), anti$\mathrm{C} 5$, anti-C5a, löslicher Komplementrezeptor 1 (sCR1) und C5-Rezeptor Antagonist (C5 RA). Gentransfer: Humaner decay accelerating factor $\left(\mathrm{DAF}^{\mathrm{g}}\right)$, membrane cofactor protein (MCP9) oder CD599. 
zeigen, daß der C6- Defekt zu einer signifikant verzögerten Bakterienelimination aus dem Blut führt (Abb. 2). Die bakterielle Kolonisierung von Leber, Milz und Lunge war bei den C6defekten Tieren am Versuchsende (180 min nach E.coli Gabe) ebenfalls verstärkt (Abb. 3; $p<0,05$ ). Diese Befunde gingen einher mit einer reduzierten Granulozyten-Burstaktivität bei den C6-defekten Tieren, während die Phagozytosekapazität unbeeinflußt blieb. Damit kann die verzögerte Bakterienelimination bei den C6-defekten Tieren unter Endotoxinämie teilweise durch die gestörte intrazelluläre Erregerabtötung erklärt werden. Da bei den untersuchten C6-defekten Tieren die C3aund C5a-Freisetzung normal abläuft, unterstreichen die erhobenen Befunde die Bedeutung des terminalen Komplementkomplexes (C5b-9) für eine erfolgreiche Erregerabwehr.

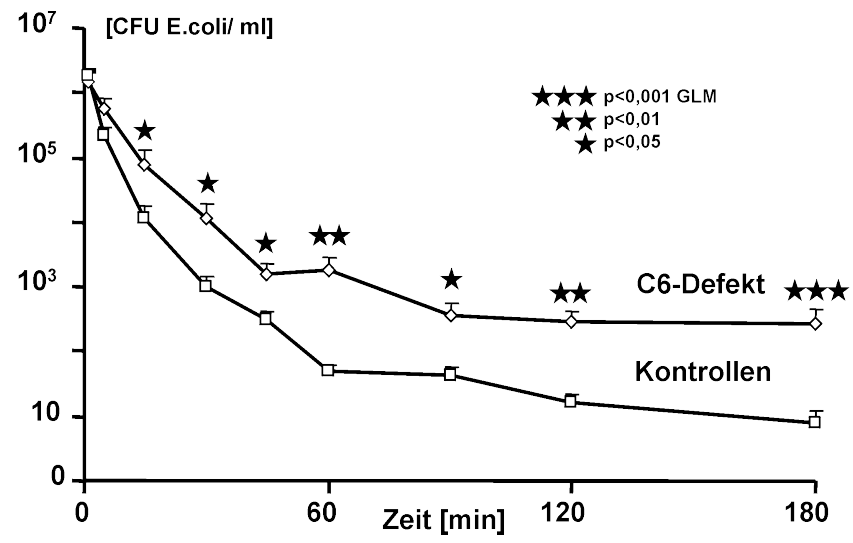

Abb. 2 Anzahl koloniebildender Einheiten (CFU; Mittelwerte \pm SEM) von Escherichia (E.) coli im Blut (CFU/ ml) nach Injektion von $10^{8} \mathrm{CFU} E$. coli in C6-defekten Tieren $(n=7)$ und in Kontrolltieren ohne C6-Defekt $(n=7)$. C6-defekt vs. Kontrolle: $p_{\text {gesamt }}=0,004 \mathrm{GLM}$.

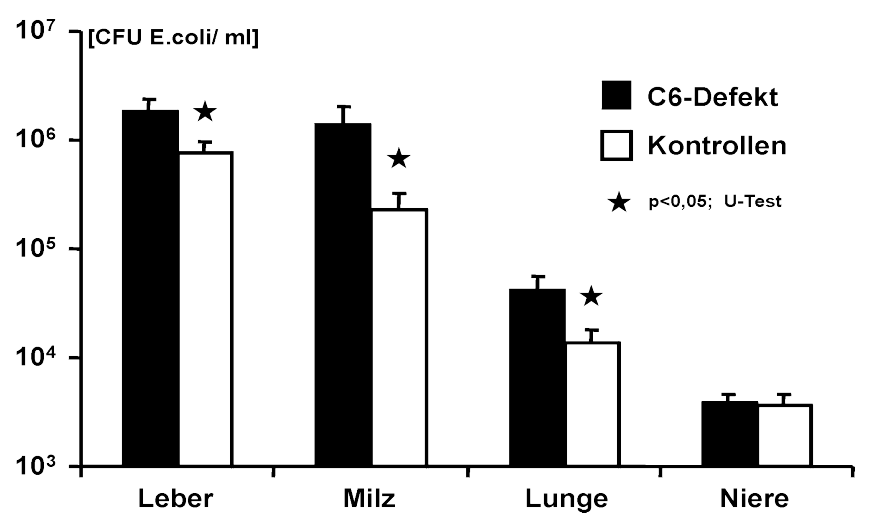

Abb. 3 Organverteilung koloniebildender Einheiten (CFU; Mittelwerte \pm SEM) von Escherichia (E.) coli 180 min. nach Injektion von $10^{8}$ CFU E. coli in C6-defekten Tieren und in Kontrolltieren ohne C6-Defekt.

Aufgrund der komplexen Interaktionen zwischen den unterschiedlichen pro-inflammatorischen Systemen stoßen Komplementspaltprodukte auch andere Entzündungskaskadensysteme an. Hieraus folgt beispielsweise die Freisetzung von toxischen Sauerstoffradikalen, Lipidmediatoren und Zytokinen. Damit kann das Komplementsystem wesentlich zu direktem oder indirektem Gewebeschaden beitragen. Unter physiologischen Bedingungen jedoch wird eine unkontrollierte Aktivie- rung von Komplement durch eine Reihe von Regulatorproteinen verhindert. Diese Regulatoren zirkulieren entweder im Plasma, wie C1-Inhibitor, oder sind auf Zelloberflächen lokalisiert. Membranständige Regulatoren wie Komplement Rezeptor 1 (CR1, CD35), Membrankofaktor Protein (MCP, CD46), „decay accelerating factor“ (DAF, CD55) oder CD59 schützen Körperzellen vor schwerwiegenden Angriffen durch das eigene Komplementsystem [11].

In den letzten Jahren sind entscheidende Fortschritte bei der Erforschung der Bedeutung des Komplementsystems bei einer Reihe von inflammatorischen Erkrankungen erzielt worden. Hierbei sind nicht nur Autoimmunerkrankungen zu nennen, sondern auch Trauma, Sepsis und Multiorganversagen [12,13]. Neben dem erweiterten Verständnis der Grundlagen der Komplementfunktion haben neuere Untersuchungen zeigen können, daß Komplementkomponenten einen prognostischen Nutzen bei der Beurteilung der Erkrankungsschwere und der frühen Identifikation von „at risk“ Patienten haben [3,4,1416]. Analysemethoden, die Anaphylatoxinmessungen im Patientenserum innerhalb von $30 \mathrm{~min}$ erlauben, könnten hier dem Intensivmediziner ein Werkzeug zur Prognoseabschätzung und Verlaufskontrolle an die Hand geben [17].

\section{Möglichkeiten der Therapeutischen Intervention}

Seit dem Ende der 60-er Jahre wird Cobra Venom Faktor (CVF) zur Untersuchung der Komplementsystems eingesetzt. CVF induziert durch Bildung einer stabilen bi-molekularen C3/C5 Konvertase eine Komplementdepletion durch Komplementverbrauch $[18,19]$. Der Wert einer Komplementdepletion für den Verlauf inflammatorischer Erkrankungen konnte mit CVF beim komplementinduzierten Lungenschaden [20,21], der allergischen Neuritis [22], Vaskulitis [23] und bei der hyperakuten Transplantatabstoßung [24] gezeigt werden. Da CVF aufgrund seiner starken Immunogenität bislang klinisch nicht eingesetzt werden konnte, wird seit seiner Sequenzierung im Jahre 1994, an rekombinanten Formen gearbeitet. Neben der Modulation des Komplementsystems durch CVF konnten bei einer Reihe von Entzündungsreaktionen günstige Effekte mit der Gabe von i.v. Immunglobulinen (IG) in supraphysiologischen Dosen erzielt werden. IGs spielen hierbei eine Rolle als Scavenger für aktiviertes C3 und C4, wobei die stärkste inhibitorische Aktivität durch ein IG-Gemisch unterschiedlicher Isotypen erzielt wurde [25]. Basta [26] beobachtete unter i.v.IG verbesserte Überlebensraten beim experimentellen Forssmann-Schock. Daneben konnte die komplementvermittelte hyperakute Transplantatabstoßung in einem Primaten Modell der Herz-Xenotransplantation verhindert werden [27]. Darüber hinaus mehren sich die Hinweise, daß die schnelle Verbesserung des klinischen Zustandsbildes bei systemischer Vaskulitis [28], dem Kawasaki Syndrom [29] oder dem bullösen Pemphigoid [30] teilweise durch einen IG-Effekt auf das Komplementsystem zu erklären sind. Immunhistologische Untersuchungen bei Dermatomyositis, die eine reduzierte C3b- und C5b-9 Bindung nach i.v.IG in den Kapillaren befallener Areale nachweisen konnten [31], unterstützen diese Befunde.

Eine weitere effektive Eingriffsmöglichkeit in die Komplementkaskade ist die Nutzung körpereigener Komplementinhibitoren, die sich durch eine hohe Spezifität und fehlende toxische Nebenwirkungen auszeichnen. C1-Inhibitor ist ein Serin Proteasen Inhibitor [32], der neben der Komplementregulation 
(C1r, C1s) auch die Kontaktaktivierung des Gerinnungssystems (Faktoren XI und XII) und des Kallikrein-Kininsystems kontrolliert [33]. C1-Inhibitor wird seit langem für die Therapie des hereditären Angioödems eingesetzt [34]. Auf der Annahme fußend, daß es bei Sepsis und SIRS durch einen relativen C1Inhibitormangel zu schwerwiegenden Komplikationen kommt, wurden unterschiedliche Untersuchungen über die Effekte einer C1-Inhibitor Substitution auf das klinische Outcome durchgeführt. Dabei konnte eine Verminderung der Komplementaktivierung bei Patienten mit septischem Schock oder kapillärem Leck nach IL-2 Therapie [35] ebenso wie eine Verbesserung des kapillären Lecks nach Knochenmarktransplantation [36] oder kindlichen Herzoperationen gezeigt werden, die zu einer Verbesserung der hämodynamischen und pulmonalen Situation führten [37]. Obwohl C1-Inhibitor im wesentlichen auf den klassischen Komplementweg wirkt, eröffnen seine gleichzeitigen Effekte auf die Gerinnung besonders in solchen Situationen Vorteile, in denen die Aktivierung beider Systeme mit einer Verschlechterung des klinischen Outcomes einher geht [38]. Zur differenzierten Untersuchung der Effekte von C1-Inhibitor auf die pulmonale Gefäßregulation haben wir in unserer Arbeitsgruppe Experimente durchgeführt [39], auf deren Ergebnisse im Verlaufe dieser Übersicht noch näher eingegangen werden wird.

Neben dem Einsatz von C1-Inhibitor kann die Komplementaktivierung auch mit Hilfe des Komplementrezeptors 1 (CR1, CD35) moduliert werden. CR1 ist ein membrangebundenes Glycoprotein das einerseits die Phagozytose von C3b-opsonierten Zielzellen mediiert, andererseits aber auch ein potenter Regulator für die C3- und die C5-Aktivierung ist [40]. Es konnte gezeigt werden, daß alleine der extramembranöse Teil des CR-1 Moleküls auch die volle regulatorische Aktivität besitzt. Dabei blockiert löslicher (s) CR-1 sowohl den klassischen als auch den alternativen Aktivierungsweg in nanomolaren Konzentrationen [41]. Nachdem die Applikation von sCR-1 sowohl in unterschiedlichen Entzündungsmodellen [39,42,43] als auch beim Menschen $[44,45]$ anti-inflammatorische Effekte gezeigt hat, befindet es sich derzeit in den USA in einer Phase II-Studie bei ARDS, Myokardinfarkt und beim Reperfusionsschaden nach Lungentransplantation.

Experimentelle Untersuchungen unserer Arbeitsgruppe nach Komplement-induziertem Lungenschaden zeigten protektive Effekte, sowohl nach der Applikation von sCR1 (TP10, Avantimmune, Needham, MA, USA) als auch nach Gabe von C1 Inhibitor (Berinert HS, Centeon, Marburg) [39]. Diese Experimente wurden an isoliert perfundierten und ventilierten Kaninchenlungen durchgeführt $[46,47]$, die eine Analyse der pulmonalen Mediatorausschüttung und der Gefäßantwort erlauben [48]. Dieses System ist zwar artifiziell, eröffnet jedoch den entscheidenden Vorteil, daß die Komplementaktivierung unter kontrollierten Bedingungen induziert werden kann, und daß die Effekte der Komplementaktivierung in Abwesenheit von Blutzellen detailliert untersucht werden können. Um zu untersuchen, ob C1-Inhibitor oder SCR1 in der Lage sind, sowohl eine pulmonale Hypertonie zu unterdrücken, als auch einer Lungenödementwicklung vorzubeugen wurden dem Perfusat 35\% humanes Serum zugefügt. Kaninchenzelloberflächen aktivieren spontan humanes Komplement, überwiegend über den alternativen Weg [49]. Hierdurch wird ein Leukozytenunabhängiger Lungenschaden induziert [50]. C1-Inhibitor $(1,0 \mathrm{U} / \mathrm{ml})$ und SCR-1 $(2,0 \mu \mathrm{g} / \mathrm{ml})$ konnten im Vergleich zur Kontrollgruppe die pulmonalvaskuläre Druckantwort reduzieren. Daneben war in diesem Modell die Lungenödembildung signifikant reduziert (Abb.4). Durch immunhistologische Anfärbung der Lungenpräparate konnten wir zeigen, daß durch Komplementmodulation im Gewebe gebundenes $\mathrm{C} 3 \mathrm{c}$ und $\mathrm{C} 5 \mathrm{~b}-9$ reduziert war (Abb. 5). Dies könnte zusammen mit den verminderten Thromboxan $\mathrm{A}_{2}$ Spiegeln nach C1-Inhibitor und sCR1 die reduzierte Druckantwort nach Komplementaktivierung erklären. Faßt man die Ergebnisse dieser und der bereits erwähnten Studien in anderen Experimentalmodellen und am Menschen zusammen, ergeben sich durch die Modulation des Komplementsystems mit körpereigenen Regulatoren wie C1-Inhibitor oder sCR1 therapeutische Optionen bei Krankheitsbildern mit starker Komplementaktivierung.

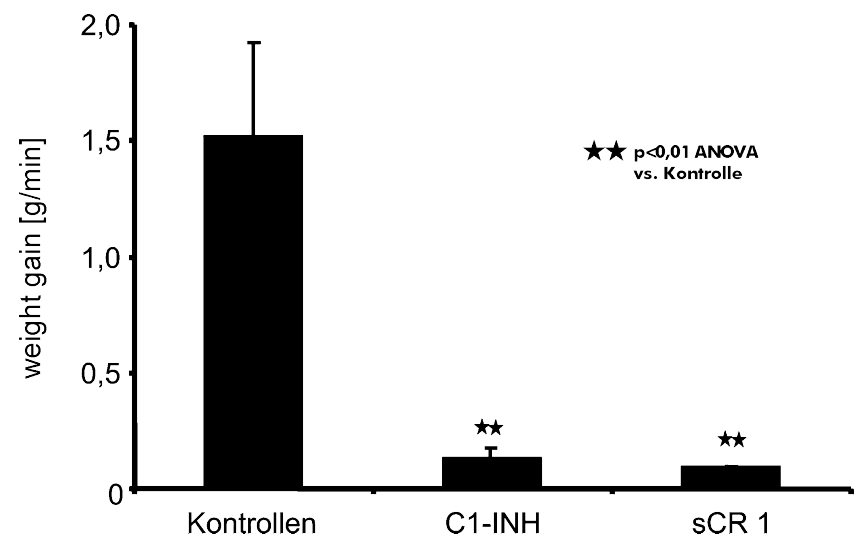

Abb. 4 Lungenödembildung (Mittelwerte \pm SEM) über den Beobachtungszeitraum in isoliert perfundierten und ventilierten Kaninchenlungen nach Komplementaktivierung ohne (Kontrollen; $n=6$ ) und mit Komplementmodulation durch $\mathrm{C} 1$-Inhibitor $(\mathrm{C} 1-\mathrm{INH} ; 1,0 \mathrm{U} / \mathrm{ml} ; \mathrm{n}=6)$ oder löslichem Komplementrezeptor 1 (sCR1; $2,0 \mu \mathrm{g} / \mathrm{ml} ; \mathrm{n}=6)$.

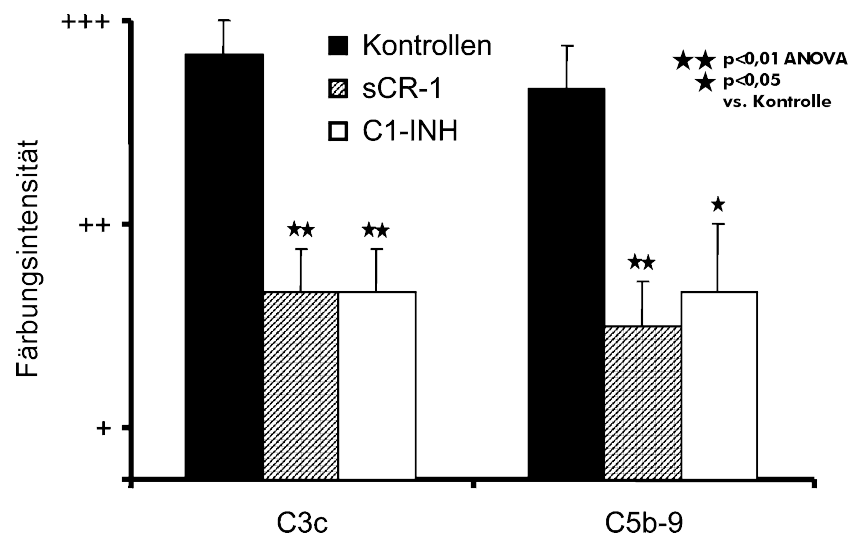

Abb. 5 Färbungsintensität von C 3 c in C 5 b-9 in isoliert perfundierten und ventilierten Kaninchenlungen nach Komplementaktivierung ohne (Kontrollen) und mit Komplementmodulation durch C1-Inhibitor (C1INH; 1,0 U/ml) oder löslichem Komplementrezeptor 1 (sCR1; 2,0 $\mu \mathrm{g} /$ $\mathrm{ml})$. Starke Färbung $(+++)$, deutliche Färbung $(++)$, gerade erkennbar $(+)$.

Neben der Modulation der Komplementkaskade durch körpereigene Regulatorproteine gibt es unterschiedliche Ansätze Komplementkomponenten zu blockieren. Dabei konnte in einer klinischen Phase I Studie gezeigt werden, daß die 
Applikation von C5-Antikörpern die Komplementaktivierung während kardiopulmonalem Bypass reduzierte [51]. Bezüglich der Blockade der Wirkung des Anaphylatoxins C5a, einem der stärksten Entzündungsmediatoren [8], gibt es in der Literatur widersprüchliche Angaben. Während in zwei Schockmodellen verbesserte Überlebensraten nach anti C5a-Antikörpergabe beobachtet wurden [52,53] konnte HÖPGEN lediglich eine Herabregulation von IL-6 ohne Verbesserung des Outcomes nachweisen [54]. Neuere experimentelle Arbeiten unterstreichen allerdings den potentiellen klinischen Nutzen der C5aInhibition [55,56]. C5a-neutralisierende Antikörper [57], C5aRezeptorantikörper [58] oder Inhibitoren der C5a-Rezeptorbindung [59] könnten damit geeignet sein, den pro-inflammatorischen Einfluß dieses Anaphylatoxins zu reduzieren. Untersuchungen mit Antikörpern gegen die Komplementrezeptoren CR3 und CR4 (CD18/CD11b,c), die die Adhäsion von Leukozyten am vaskulären Endothel vermitteln, zeigen in ihrer antiinflammatorischen Wirkung ein einheitlicheres Bild. So wurde durch frühe Applikation von anti-CD11b eine erhöhte Überlebensrate bei experimenteller Encephalitis [60] und myokardialem Reperfusionsschaden [61] nachgewiesen.

Weitere potentielle Indikationen für Komplementinhibitoren ergeben sich bei Transplantationen. Seit vielen Jahren stellt der Mangel an Spenderorganen ein zentrales Problem in der Transplantationsmedizin dar [62]. Ein möglicher Ausweg aus dieser Situation könnte die Verwendung von xenogenen Spenderorganen sein. Zur Bekämpfung der Komplementinduzierten hyperakuten Transplantatabstoßung wurden bei Xenotransplantationen humane Komplementregulatorproteine erfolgreich auf xenogenes Gewebe transferiert. Dalmasso [63] konnte zeigen, daß humaner DAF in Endothelzellmembranen von Schweinen eingebaut werden kann und damit die Lyse durch humanes Komplement verhindert. Auch die Transfektion von Schweineendothelzellen mit humanem CD59 verhinderte die Komplement vermittelte Zellzerstörung [64]. Ein wesentlicher Fortschritt für Xenotransplantationen war die Erzeugung transgener Spendertiere, die humane Komplementregulatorproteine in ihrem Gewebe exprimieren [65] und nach Xenoperfusion geringere Organzeichen der hyperakuten Transplantatabstoßung zeigten [66,67]. Durch die Überwindung der Komplementinduzierten Abstoßungsreaktion bei Xenotransplantationen könnten in Zukunft Engpässe bei der Versorgung mit Spenderorganen beseitigt werden. Potentielle infektiologische Probleme bei Xenotransplantationen, die auf noch nicht abschließend geklärte Interaktionen zwischen humanen und porcinen Retroviren nach der Transplantation zurückzuführen sind, müssen jedoch zunächst geklärt werden [62].

\section{Ausblick}

Ermutigt durch neue experimentelle Befunde und erste klinische Ergebnisse werden verschiedene Strategien der Komplementmodulation zum Einsatz bei inflammatorischen Erkrankungen propagiert. Obwohl eine effektive Komplementinhibition beispielsweise die Voraussetzung für erfolgreiche Xenotransplantationen ist, muß berücksichtigt werden, daß eine systemische Komplementinhibition den Patienten eines wichtigen Armes der immunologischen Abwehr beraubt.

Die Kenntnisse über den zeitlichen Verlauf der Komplementaktivierung, über das Ineinandergreifen verschiedenster Pa- thomechanismen bei pathogenetisch unterschiedlichen inflammatorischen Prozessen und nicht zuletzt über patientenspezifische Dispositionen konnten in den letzten Jahren durch zahlreiche experimentelle und klinische Studien erweitert werden. Die Komplexität schwerer inflammatorischer Erkrankungen läßt jedoch eine monokausale Therapie septischer Komplikationen unwahrscheinlich erscheinen. Weitere Studien müssen klären, inwieweit Eingriffe in das Komplementsystem Morbidität und Mortalität bei kritisch Kranken senken können. Durch die vielfältigen Effekte des Komplementsystems im Rahmen von Immunabwehr und Inflammation scheint die differenzierte Analyse des Immunstatus von besonderer Bedeutung zu sein, um in Zukunft eine bedarfsadaptierte antiinflammatorische Therapie durchführen zu können.

\section{Literatur}

${ }^{1}$ Bauer M, Rensing H, Ziegenfuß T: Anästhesie und perioperative Immunfunktion. Anaesthesist 1998; 47: 538 - 556

${ }^{2}$ Brand JM, Kirchner H, Poppe C, Schmucker P: Zytokinfreisetzung und Verteilung mononukleärer Zellen im peripheren Blut unter dem Einfluß der Allgemeinnarkose. Anaesthesist 1998; 47: 379 386

${ }^{3}$ Gama de Abreu M, Kirschfink M, Quintel M, Albrecht DM: White blood cell counts and plasma C3a have synergistic predictive value in patients at risk for acute respiratory distress syndrome. Crit Care Med 1998; 26: 1040 - 1048

${ }^{4}$ Zilow G, Sturm JA, Rother U, Kirschfink M: Complement activation and the prognostic value of $\mathrm{C} 3 \mathrm{a}$ in patients at risk of adult respiratory distress syndrome. Clin Exp Immunol 1990; 79: 151 157

${ }^{5}$ Müller-Eberhard HJ: Molecular organization and function of the complement system. Ann Res Biochem 1988; 57: 321 - 347

${ }^{6}$ Rother K, Till GD: The Complement System. Springer. Berlin/ Heidelberg/New York. 1988

${ }^{7}$ Turner MW: Mannose-binding lectin: The pluripotent molecule of the innate immune system. Immunol Today 1996; 17: 532 - 540

${ }^{8}$ Hugli TE: Biochemistry and biology of anaphylatoxins. Complement 1986; 3: $111-127$

${ }^{9}$ Rieber EP: Aktivierung von Lymphozyten durch Antigen. In: Baenkler HW (Hrsg.) Medizinische Immunologie, Ecomed 1995: $113-135$

${ }^{10}$ Heller A, Koch T, Kirschfink M: Significance of the terminal complement complex, C5b-9, in bacterial clearance and neutrophil function. Immunobiology 1999; 200: 627 (Abstract)

${ }^{11}$ Devine DV: The regulation of complement on ceII surfaces. Transfusion Med Rev 1991; V: 123 - 131

${ }^{12}$ Dalmasso AP: Complement in the pathophysiology and diagnosis of human diseases. Crit Rev Clin Lab Sci 1986; 24: 123 - 183

${ }^{13}$ Morgan BP: Complement Clinical Aspects and Relevance to Disease. Harcourt Brace Jovanovich London; New York 1990

${ }^{14}$ Kirschfink M, Wienert T, Rother K, Poiner S: Complement activation in renal allograft recipients. Transpl Proc 1992; 24: 2556 2557

${ }^{15}$ Hecke F, Schmidt U, Kola A, Bautsch W, Klos A, Kühl J: Analysis of complement proteins in polytrauma patients-correlation with injury, severity, sepsis and outcome. Shock 1997; 7: 74

${ }^{16}$ Stöve S, Welte T, Wagner TOF, Kola A, Klos A, Bautsch W, Köhl J: Circulating complement proteins in patients with sepsis or systemic inflammatory response syndrome. Clin Diagn Lab Immunol 1996; 3: $175-183$

${ }^{17}$ Hartmann H, Lübbers B, Casaretto M, Bautsch W, Klos A, Köhl J: Rapid quantification of $\mathrm{C} 3 \mathrm{a}$ and $\mathrm{C} 5 \mathrm{a}$ using a combination of chromatographic and immunoassay procedures. J Immunol Methods 1993; 166: $35-44$ 
${ }^{18}$ Cochrane CG, Müller-Eberhard HJ, Aikin BS: Depletion of plasma complement in vivo by a protein of cobra venom. Its effect on various immunologic reactions. J Immunol 1970; 105: 55 - 69

${ }^{19}$ Fritzinger DC, Bredehorst R, Vogel CW: Molecular cloning and derived primary structure of cobra venom factor. Proc Natl Acad Sci USA 1994; 91: 12775 - 12779

${ }^{20}$ Ren XD, Huang SJ, Sun JJ, Zhu ZG: Protective effect of cobra venom factor on pulmonary injury induced by oleic acid. Int J Immunopharmacol 1994; 16: 969 - 975

21 Dehring DJ, Seinberg SM, Wismar BL, Lowery BD, Carev LZ, Cloutier CT: Complement depletion in a porcine model of septic acute respiratory disease. J Trauma 1987; 27: $615-625$

${ }^{22}$ Vriesendorp FJ, Flynn RE, Pappolla MA, Koski CL: Complement depletion affects demyelination and inflammation in experimental allergic neuritis. J Neuroimmunol 1995; 58: 157 - 165

${ }^{23}$ Mathieson PW, Qasim FJ, Thiru S, Oldroyd RG, Oliveira DB: Effects of decomplementation with cobra venom factor on experimental vasculitis. Clin Exp Immunol 1994; 97: 474 - 477

${ }^{24}$ Azimzadeh A, Wolf P, Dalmasso AP, Odeh M, Beller JP, Fabre M, Charreau B, Thibaudeau K, Cinqualbre J, Soulillou JP, Anegon I: Assesment of hyperacute rejection in a rat-to-primate cardiac xenograft model. Transplantation 1996; 61: $1305-1313$

${ }^{25}$ Miletic VD, Hester CG, Frank MM: Regulation of complement activity by immunoglobulin. Effect of immnunoglobulin isotype on C4 uptake on antibody sensitized sheep erythrocytes and solid phase immune complexes. J Immunol 1996; 156: 749 - 757

${ }^{26}$ Basta M, Kirschbom P, Frank MM, Fries LF: Mechanism of therapeutic effect of high-dose intravenous immunoglobulin. Attenuation of acute complement-dependent immune damage in a guinea pig model. J Clin Invest 1989; 84: 1974 - 1981

${ }^{27}$ Magee JC, Collins BH, Harland RC, Lindman BJ, Bollinger RR, Frank MM, Platt JL: Immunoglobulin prevents complement-mediated hyperacute rejection in swine-to-primate xenotransplantation. J Clin Invest 1995; 96: $2404-2412$

${ }^{28}$ Gross WL: New developments in the treatment of systemic vasculitis. Curr Opin Rheumatol 1994; 6: $11-19$

${ }^{29}$ Newburger JW, Takahashi M, Burns JC, Beiser AS, Chung KJ, Duffy CE: The treatment of Kawasaki syndrome with intravenous gamma globulin. N Engl J Med 1986; 315: 341 - 347

${ }^{30}$ Stiehm ER, Ashida E, Kim KS, Winston DJ, Haas A, Gale RP: Intravenous immunoglobulins as therapeutic agents (clinical conference). Ann Int Med 1987; 107: $367-382$

${ }^{31}$ Basta M, Dalakas MC: High-dose intravenous immunoglobulin exerts beneficial effect in patients with dermatomyositis by blocking endomysial deposition of activated complement fragments. J Clin Invest 1994; 94: 1729 - 1735

${ }^{32}$ Carrell RW, Boswell DR, Serpins: The superfamily of plasma serine protease inhibitors. In: Barret AJ, Salvese G (Eds.) Proteinase Inhibitors Elsevier Amsterdam 1986: 403 - 420

${ }^{33}$ Schapira M, Agostoni de A, Schifferli JA, Colmnan RW: Biochemistry and pathophysiology of human $\mathrm{C} 1$ inhibitor. Current issues Complement 1985; 2: 111 - 126

${ }^{34}$ Gadek JE, Hasea JA, Santaella M, Wickerhausen M, Triantaphyllopoulos DC, Frank MM: Replacement therapy in hereditary angioedema. Successful treatment of acute episodes of angioedema with partly purified C1 inhi-bitor. N Engl J Med 1980; 302: $542-546$

${ }^{35}$ Hack CE, Ogilvies AC, Eisele B, Eerenberg AJM, Wagstaff J, Thijs LG: C1-inhibitor substitution therapy in septic shock and in the vascular leak syndrome induced by high doses of interleukin-2. Intensive Care Med 1993; 19: 19 - 28

${ }^{36}$ Nürnberger W, Göbel U: Capillary leak syndrome following bone marrow transplantation. Biomed Progr 1996; 9: 8 - 11

${ }^{37}$ Buerke M, Murohara T, Lefer AM: Cardioprotective effects of a C1 esterase inhibitor in myocardial ischemia and reperfusion. Circulation 1995; 91: $393-402$
${ }^{38}$ Nuijens JH, Huijbregts CC, Eerenberg-Belmer AJ, Abbink JJ, Strak van Schijndel RJ, Felt-Bersma RJ, Thijs LG, Hack CE: Quantification of plasma factor XIIa-C1-inhibitor and kallikrein-C1-inhibitor complexes in sepsis. Blood 1988; 72 : $1841-1848$

${ }^{39}$ Heller A, Kunz M, Samakas A, Haase M, Kirschfink M, Koch T: The complement regulators $C 1$ inhibitor and soluble complement receptor 1 attenuate acute lung injury in rabbits. Shock (zur Publikation angenommen)

${ }^{40}$ Fearon DT: Regulation of the amplification C3 convertase of human complement by an inhibitory protein isolated from human erythrocyte membranes. Proc Natl Acad Sci USA 1979; 76: 5867 5871

${ }^{41}$ Fearon DT: Anti-inflammatory and immunosuppressive effects of recombinant soluble complement receptors. Clin Exp Immunol 1991; 86: $43-46$

${ }^{42}$ Homeister JW, Lucchesi BR: Complement activation and inhibition in myocardial ischemia and reperfusion injury. Annu Rev Pharmacol Toxicol 1994; 34: 17 - 40

${ }^{43}$ Chavez Cartaya RE, DeSola GP, Wright L, Jamieson NV, White DJ: Regulation of the complement cascade by soluble complement receptor type 1 . Protective effect in experimental liver ischemia and reperfusion. Transplantation 1995; 59: 1047 - 1052

${ }^{44}$ Rabenovici R, Yeh CG, Hillegass LM, Griswold DE, DiMartino MJ, Vernick J, Fong KLL, Feuerstein G: Role of complement in endotoxin platelet activating factor induced lung injury. J Immunol 1992; 149: 1744 - 1750

${ }^{45}$ Couser WG, Johnson RJ, Young BA, Yeh CG, Toth CA, Rudolph AR: The effects of soluble recombinant complement receptor 1 on complement-mediated experimental glomerulonephritis. J Am Soc Nephrol 1995; 5: $1888-1894$

${ }^{46}$ Heller A, Fiedler F, Schmeck J, Lück V, Iovanna JL, Koch T: Pancreatitis associated protein protects the lung from leukocyte induced injury. Anesthesioloy 1999; 91(5): (im Druck)

${ }^{47}$ Heller A, Koch T: Pharmakologische Aspekte von mehrfach ungesättigten Fettsäuren in der parenteralen Ernährung. Anästhesiol Intensivmed Notfallmed Schmerzther 1998; 33: $77-87$

${ }^{48}$ Seeger W, Walmrath D, Grimminger F: Adult Respiratory Distress Syndome: Model systems using isolated perfused rabbit lungs. Meth Enzym 1994; 233: 549 -584

${ }^{49}$ Platt-Mills TAE, Ishizaka K: Activation of the alternate pathway of human complement by rabbit cells. J Immunol 1974; 113: 348 358

${ }^{50}$ Seeger W, Hartmann R, Neuhof H, Bhakdi S: Local complement activation, thromboxane-mediated vasoconstriction, and vascular leakage in isolated lungs. Am Rev Respir Dis 1989; 139: 88 -99

${ }^{51}$ Rollins SA, Rinder HM, Rinder CS, Fitch JCK, Smith BR, Hines RL, Alford BL, Matis LA: A humanized anti-C5 scFv blocks platelet and leukocyte activation and exhibits prolonged pharmacokinetics and pharmacodynamics in humans. Exp Clin immunogenet 1997; 14: 36

52 Stevens JH, O'Hanley P, Shapiro JM, Mihm FG, Satoh PS, Collins JA, Raffin TA: Effects of anti-C5a antibodies on the adult respiratory distress syndrome in septic primates. J Clin Invest 1986; 77: $1812-1816$

${ }^{53}$ Smedegard G, Cui L, Hugli TE: Endotoxin induced shock in the rat. A role for C5a. Am J Pathol 1989; 135: $489-479$

${ }^{54}$ Höpgen U, Mohr M, Struber A, Montz H, Buchardi H, Götze O, Oppermann M: Inhibition of Interleukin-6 synthesis in an animal model of septic shock by anti-C5a monoclnal antibodies. Eur J Immunol 1996; 26: 1103 - 1109

${ }^{55}$ Park KW, Tofukuji M, Metais C, Comunale ME, Dai HB, Simons M, Stahl GL, Agah A, Sellke FW: Attenuation of endotheliumdependent dilation of pig pulmonary arterioles after cardiopulmonary bypass is prevented by monoclonal antibody to complement C5a. Anesth Analg 1999; 89: 42 - 48 
${ }^{56}$ Czermak BJ, Sarma V, Pierson CL, Warner RL, Huber-Lang M, Bles NM, Schmal H, Friedl HP, Ward PA: Protective effects of C5a blokkade in sepsis. Nat Med 1999; 5: $788-792$

${ }^{57}$ Vakeva AP, Agah A, Rollins SA, Matis LA, Li L, Stahl GL: Myocardial infarction and apoptosis after myocardial ischemia and reperfusion: role of the terminal complement components and inhibition by anti-C5 therapy. Circulation 1998; 97: $2259-2267$

${ }^{58}$ Morgan EL, Ember JA, Sanderson SD, Scholz W, Buchner R, Ye RD, Hügli TE: Anti-C5a receptor antibodies. Characterization of neutralizing antibodies specific for a peptide, C5aR-(9-29), derived from the predicted amino-terminal sequence of the human C5a receptor. J Immunol 1993; 151: 377 - 388

${ }^{59}$ Lanza TJ, Durette PL, Rollins T, Siciliano S, Cianciarulo DN, Kobayashi SV, Caldwell CG, Springer MS, Hagmann WK: Substituted 4,6-diaminoquinolines as inhibitors of C5a receptor binding. J Med Chem 1992; 35: 252 - 258

${ }^{60}$ Huitinga I, Damoiseaux JGMC, Döpp EA, Dijkstra CD: Treatment with anti-CR-3 antibodies ED7 and ED8 supperses experimental allergic encephalomyelitis in Lewis rats. Eur J. Immunol 1993; 23: $709-715$

${ }^{61}$ Simpson J, Todd RF III, Fantone JC, Mickelson JK, Griffin JD, Luccresi BR, Adams D, Hoff P, Leeh K, Rogers CE: Reduction of experimental canine reperfusion injury by a monoclonal antibody (anti-Mo1, anti CD11b) that inhibits leukocyte adehsion. J Clin Invest 1988; 81: $624-629$

${ }^{62}$ Schlitt HJ, Manns MP: Ethische und rechtliche Aspekte der Xenotransplantation. Dt Ärztebl 1999; 96: B1473 - B1476

${ }^{63}$ Dalmasso AP, Vercellotti GM, Platt JL, Bach FH: Inhibition of complement-mediated endothelial cell cytotoxicity by decayaccelerating factor. Transplantation 1991; 52: 530 - 533

${ }^{64}$ Heckl-Östreicher B, Wosnik B, Kirschfink M: Protection of porcine endothelial cells from complement-mediated cytotoxicity by the human complement regulators CD59 C1 inhibitor and soluble complement receptor 1 . Analysis in a pig-to-human in vitro model relevant to hyperacute rejection. Transplantation 1996; 62: $1-3$

${ }^{65}$ Cozzi E, White DJ: The generation of transgenic pigs as potential Organ donors for humans. Nat Med 1995; 1: 964 - 966

${ }^{66}$ Schmöckel M, Nollert G, Shahmohammadi M, Young VK, Knig W, White DJ, Hammer C, Reichart B: Human decay accelerating factor successfully protects pig hearts from hypcracute rejection by human blood. Transplant Proc 1996; 28: 768 - 769

${ }^{67}$ Byrne GW, McCurry KR, Martin MJ, McClellan SM, Platt JL, Logan JS: Transgenic pigs expressing human CD59 and decay accelerating factor produce an intrinsic barrier to complement mediated damage. Transplantation 1997; 63: 149- 155

\section{Dr. Axel Heller}

Klinik und Poliklink für Anaesthesiologie und Intensivmedizin Universitätsklinikum Carl Gustav Carus

Technische Universität Dresden

Fetscherstraße 74

01307 Dresden

E-mail: heller-a@rcs.urz.tu-dresden.de WEB: http://www.tu-dresden.de/medkai/ 